Please do not remove this page

RMIT

UNIVERSITY

\title{
Performance of STF coded OFDM with transmit eigenbeamforming in correlated fading channels
}

Lin, Kevin; Hussain, Zahir

https://researchrepository.rmit.edu.au/esploro/outputs/9921862783301341/filesAndLinks?institution=61RMIT_INST\&index=null

Lin, K., \& Hussain, Z. (2006). Performance of STF coded OFDM with transmit eigenbeamforming in correlated fading channels. 12th Asia Pacific Conference on Communications, 1-5.

https://doi.org/10.1109/APCC.2006.255778

Published Version: https://doi.org/10.1109/APCC.2006.255778

Repository homepage: https://researchrepository.rmit.edu.au

(c) 2006 IEEE. Personal use of this material is permitted. However, permission to reprint/republish this material for advertising or promotional purposes or for creating new collective works for resale or redistribution to servers or lists, or to reuse any copyrighted component of this work in other works must be obtained from the IEEE.

Downloaded On 2023/04/27 01:12:39+1000 


\title{
Performance of STF Coded OFDM with Transmit Eigenbeamforming in Correlated Fading Channels
}

\author{
Kevin H. Lin and Zahir M. Hussain \\ School of Electrical and Computer Engineering, RMIT University \\ 124 LaTrobe Street, Melbourne, Victoria 3000, Australia \\ Emails: s9510490@student.rmit.edu.au; zmhussain@ieee.org
}

\begin{abstract}
A new transmit diversity configuration that combines transmit eigenbeamforming with space-time-frequency (STF) coding for orthogonal frequency division multiplex (OFDM) systems is imestigated. Based on the signal angleof-arrival (AoA) estimates, channel correlation matrix is constructed. It is shown that signal transmission of STF codes in the eigen-modes of this matrix gives an effective array weighting gain which improves system error performance without sacrificing any diversity and coding gain. For imperfect AoA estimation, error probability loss due to incorrect estimation is quantified and the required accuracy of AoA estimation for securing the performance gain is shown for different angular spreads.
\end{abstract}

\section{Introduction}

Driven by growing demand in high speed mobile services, space-time coding (STC) [1] and [2] have attracted considerable attention due to the benefits of diversity and coding gains. The application of STC to OFDM systems was first introduced in [3] to achieve high data-rate transmissions for delay-sensitive services. Motivated by the additional diversity branches in broadband multipath channels, space-frequency (SF) and STF coding that exploits space and multipath diversity is proposed in [4]-[6]. However, their maximum performance gain is only achievable in spatial uncorrelated channels. Studies in [6]-[8] have shown that the performance of space-time codes and the capacity of multi-antenna systems degrade significantly in the presence of fading correlation. Combining coding with linear processing at the transmitter and the receiver, [9] showed that wireless transmission can be transformed into a set of parallel scalar channels and channel capacity is maximized in multipath environment.

In this work, we introduce an adaptive transmission scheme that jointly uses transmitter eigenbeamforming and STF coding (Eigen-STF) to provide an array weighting gain (AWG) and preserve the diversity and coding gains offered by the STF coding scheme for broad-band OFDM systems. Based on uplink AoA estimates, spatial correlation structure of the propagation channel is first estimated at the base station (BS), then STF encoded information is transmitted in the eigen-directions of this channel and lead to uncorrelated fading for each antenna. The resultant effect is an enhancement of SNR at the mobile station (MS); this gives an improvement in system error-rate performance. The proposed solution depends only on the channel correlation matrix, and thus can be applied without any feedback mechanism or channel reciprocity assumption. This makes it more practical as compared to the traditional beamforming schemes where output beam resolution can suffer greatly from channel estimation, quantization, and feedback errors and delay. In this work, we also analyze the effects of imperfect AoA estimation to justify the performance gain of the proposed structure.

Notation used: $(.)^{*},(.)^{T}$, and $(.)^{H}$ are conjugation, transposition, and Hermitian transposition, respectively. $\|\mathcal{A}\|_{F}, \sqrt{\mathcal{A}}$ and $\operatorname{det}(\mathcal{A})$ denote the Frobenius norm, Hermitian square root, and the determinant of matrix $\mathcal{A}$, respectively. Capital bold letters represent matrices, small underlined are vectors.

\section{System Model}

A general structure of a MIMO-OFDM system employing the proposed Eigen-STF transmission structure is illustrated in Fig. 1 with $N_{t}$ transmit and $N_{r}$ receive antennas. The OFDM system we consider here uses $N_{c}$ frequency tones and the channel is assumed to be frequency-selective.

At the BS, STF encoding is performed by formatting a sequence of baseband modulated data symbols into a codeword matrix $\mathcal{C}:=\left[\mathbf{C}_{1}, \cdots, \mathbf{C}_{k}, \cdots, \mathbf{C}_{N_{c}}\right] \in \mathbb{C}^{N_{t} \times p N_{c}}$, where $\mathbf{C}_{k}$ is a $N_{t} \times p$ sub-matrix (which spans across $p$ 


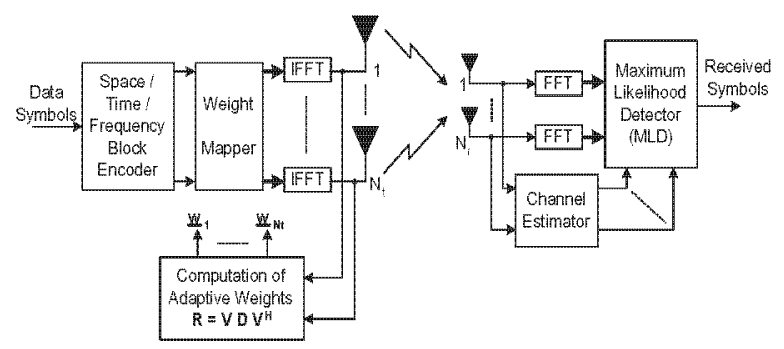

Figure 1. General structure of STF-OFDM system with adaptive weighting scheme. Bold arrows represent multi-line signals.

OFDM-symbol intervals and $N_{t}$ transmit antennas) to be transmitted on the $k^{\text {th }}$ subcarrier. Because the number of baseband constellation points is finite, there is a limited number of possible STF codeword matrices and we denote this finite set as $\Upsilon \ni \mathcal{C}$. In this letter, details of STF construction are not described as we consider only the performance of the proposed transmission structure, not the codeword itself. We refer readers to [4] and [5] for detailed description of design criteria and the formatting of STF codewords.

\subsection{Channel Model}

Consider a typical cellular communication scenario where the BS antennas are placed at the building roof-top in an unobstructed environment and the MS is located at the street level and surrounded by dense distribution of local scatterers. It is stated in [10] that signal transmission in such an environment over a multipath channel leads to uncorrelated signal paths arriving at the MS but partially correlated in spatial domain at the BS. Assuming that a uniform linear array (ULA) configuration is used for $N_{t}$ BS antennas with a spacing of $d$ meters, the normalized transmit spatial covariance matrix that specifies the spatial correlation between antenna elements is defined as [11]

$$
\mathbf{R}_{t}=\frac{1}{L} \sum_{\ell=1}^{L} \underline{a}\left(\phi_{\ell}\right) \underline{a}^{H}\left(\phi_{\ell}\right),
$$

where $L$ denotes the number of dominant resolvable paths, $\underline{a}\left(\phi_{\ell}\right):=\left[1, e^{j \beta}, e^{j 2 \beta}, \cdots, e^{j\left(N_{t}-1\right) \beta}\right]^{T}$ is the array propagation vector for the $\ell^{\text {th }}$ tap with an AoA of $\phi_{\ell}$, and $\beta=\left[2 \pi \cdot d \cdot \sin \left(\phi_{\ell}\right)\right] / \lambda$ with $\lambda$ being the carrier frequency wavelength. In general, $\mathbf{R}_{t}$ is a nonnegativedefinite Hermitian matrix and its eigenvalue-decomposition (EVD) can be expressed as $\mathbf{R}_{t}=\mathbf{V D V}^{H}$, where $\mathbf{V}=$ $\left[\underline{v}_{1}, \cdots, \underline{v}_{N_{t}}\right]$ is a unitary matrix with columns that are the eigenvectors of $\mathbf{R}_{t}$ and $\mathbf{D}=\operatorname{diag}\left\{\mu_{1}, \mu_{2}, \cdots, \mu_{N_{t}}\right\}$ contains the corresponding eigenvalues. Define $\underline{g}_{i, j}=$ $\left[g_{i, j}(0), \cdots, g_{i, j}(L-1)\right]$ as the $L$-tap channel impulse response vector for the $(i, j)^{\text {th }}$ receive-transmit antenna pair. The channel frequency response matrix can be expressed as $\mathbf{H}_{k}$ with its $(i, j)^{\text {th }}$ entry $h_{i, j, k}=\underline{g}_{i, j} \underline{f}_{k}$ where $\underline{f}_{k}=$ $\left[1, e^{-j 2 \pi(k-1) / N_{c}}, \cdots, e^{-j 2 \pi(k-1) \tau_{L-1} / N_{c}}\right]^{T}$ is the corresponding discrete Fourier transform coefficients and $\tau_{\ell}$ is the integer delay of the $\ell^{\text {th }}$ tap. The correlated channel frequency response can then be given as $\mathbf{H}_{k} \sqrt{\mathbf{R}_{t}}$. We assume that the spatial correlation is the same for all subcarriers.

\section{$2.2 \quad$ Signal Model}

To facilitate STF codeword transmission in the eigenmodes of the correlation matrix, eigen weight mapping is performed across the space dimension of the STF codeword $\left\{\mathbf{C}_{k}\right\}_{k=1}^{N_{c}}$ prior to transmission. Mathematically, it can be expressed as $\mathbf{W}^{H} \mathbf{C}_{k}$, where $\mathbf{W}=\left[\underline{w}_{1}, \cdots, \underline{w}_{N_{t}}\right]$ is the eigen weight mapping matrix and $\underline{w}_{j}=\underline{v}_{j}$. Then signal transmission on different eigenvectors of $\mathbf{R}_{t}$ amounts for transmitting $N_{t}$ orthonormal beams in the direction of the dominant multipaths seen by the BS. In the case when $\mathbf{R}_{t}$ is not the same for all subcarriers, beamforming should be performed individually for groups of subcarriers with one coherent bandwidth apart.

At the receiver, discrete Fourier transformation (DFT) is applied to the received signals from $N_{r}$ antennas. The discrete time baseband equivalent expression of the received signal has the form

$$
\mathbf{Y}_{k}=\mathbf{H}_{k} \sqrt{\mathbf{R}_{t}} \mathbf{W}^{H} \mathbf{C}_{k}+\mathbf{E}_{k},
$$

where $\mathbf{E}_{k}$ is the receiver noise matrix and its elements are modelled as uncorrelated white Gaussian random variables having $\mathcal{N}\left(0, \sigma_{n}^{2}\right)$. At the receiver, channel estimation is performed by correlating pilot tones embedded in the transmitted signal. The result is then fed into the MLD for STF decoding of data symbols by evaluating the decision matrix as follows

$$
\widehat{\mathcal{C}}=\arg \min _{\mathcal{C} \in \boldsymbol{\Upsilon}} \sum_{k=1}^{N_{c}}\left\|\mathbf{Y}_{k}-\mathbf{H}_{k} \sqrt{\mathbf{R}_{t}} \mathbf{W}^{H} \mathbf{C}_{k}\right\|_{F}^{2} .
$$

\section{Performance Analysis}

\subsection{Pairwise Error Probability}

Assuming that the receiver has perfect knowledge of the channel and that the decision matrix in (3) is evaluated at the MLD for symbol detection, the pairwise error probability (PEP) for an erroneous detection of the codeword $\widetilde{\mathcal{C}}$ in favor of $\mathcal{C}$ conditioned on the fading channel 
$\mathcal{H}=\left\{h_{i, j, k}\right\}_{i=1, j=1, k=1}^{N_{r}} \begin{aligned} & N_{t} \\ & N_{c} \\ & k=1\end{aligned}$ can be expressed as

$$
P_{r}(\mathcal{C} \rightarrow \widetilde{\mathcal{C}} \mid \mathcal{H})=Q\left(\sqrt{\frac{d^{2}(\mathcal{C}, \widetilde{\mathcal{C}}) \rho}{2}}\right)
$$

where $\rho=\varepsilon_{s} / \sigma_{n}^{2}, \varepsilon_{s}$ is the average symbol energy, $Q(\cdot)$ is the classical Gaussian $Q$-function, and

$$
d^{2}(\mathcal{C}, \widetilde{\mathcal{C}}):=\sum_{k=1}^{N_{c}}\left\|\mathbf{H}_{k} \sqrt{\mathbf{R}_{t}} \mathbf{W}^{H}\left(\mathbf{C}_{k}-\widetilde{\mathbf{C}}_{k}\right)\right\|_{F}^{2}
$$

is commonly known as the squared Euclidean distance between the two codeword matrices. Using the Chernoff bound in [12], (4) can be upper-bounded as

$$
P_{r}(\mathcal{C} \rightarrow \widetilde{\mathcal{C}} \mid \mathcal{H}) \leq \exp \left(\frac{-d^{2}(\mathcal{C}, \widetilde{\mathcal{C}}) \rho}{4}\right)
$$

Defining $\underline{g}_{i}=\left[\underline{g}_{i, 1}, \cdots, \underline{g}_{i, N_{t}}\right] \in \mathbb{C}^{1 \times N_{t} L}$ and $\mathbf{F}_{k}:=$ $\operatorname{diag}\{f(k), \cdots, f(k)\} \in \mathbb{C}^{N_{t} L \times N_{t}}$, the squared Euclidean distance can be rewritten as $d^{2}(\mathcal{C}, \widetilde{\mathcal{C}})=\sum_{i=1}^{N_{r}} \underline{g}_{i} \Psi \underline{g}_{i}^{H}$, where $\boldsymbol{\Psi}=\sum_{k=1}^{N_{c}} \mathbf{F}_{k} \sqrt{\mathbf{R}_{t}} \mathbf{W}^{H} \mathbf{B}_{k} \mathbf{B}_{k}^{H} \mathbf{W}{\sqrt{\mathbf{R}_{t}}}^{\bar{H}} \mathbf{F}_{k}^{H} \in$ $\mathbb{C}^{N_{t} L \times N_{t} L}$ is the effective error distance between two distinct codeword matrices and $\mathbf{B}_{k}=\mathbf{C}_{k}-\widetilde{\mathbf{C}}_{k}$. It is observed that $\Psi$ is a non-negative definite Hermitian matrix, and thus, EVD of $\boldsymbol{\Psi}$ has a form of $\Lambda \Psi \Lambda^{H}=\Omega$, where $\Lambda$ is a unitary matrix, $\Omega=\operatorname{diag}\left\{\omega_{1}, \cdots, \omega_{r}\right\}$ contains nonzero eigenvalues, and $r$ is the rank of $\Psi$. We assume that the elements of $\left\{\underline{g}_{i}\right\}_{i=1}^{N_{r}}$ are independent and identically distributed (i.i.d.) complex Gaussian random variables. By averaging the conditional PEP in (5) over all channel realizations, the PEP of an Eigen-STF OFDM system can be finally written as

$$
P_{r}(\mathcal{C} \rightarrow \widetilde{\mathcal{C}}) \leq\left(\prod_{m=1}^{r} \omega_{m}\right)^{-N_{r}}\left(\frac{\gamma}{4}\right)^{-r N_{r}}
$$

where $\gamma=\rho\left\|\sqrt{\mathbf{R}_{t}} \mathbf{W}^{H}\right\|_{F}^{2}$ denotes the effective receiver SNR. Then the AWG of this system over systems without beamforming can be found as $\sum_{j=1}^{L} \underline{w}_{j} \mathbf{R}_{t} \underline{w}_{j}^{H}$.

\subsection{Diversity Order}

Based on the above error probability analysis, a few remarks are now in order. First, by checking the dimensionality of $\Psi$, the maximum diversity order in our system is $N_{t} L N_{t}$. Suppose that $\mathbf{B}$ has full rank for all pairs of matrices $\mathbf{C}_{k} \neq \widetilde{\mathbf{C}}_{k}$, which is a necessary assumption for the design of STF codeword to exploit the space diversity. If $\mathbf{R}_{t}$ has a rank of $N_{t}$, the system will achieve the maximum diversity order since the weight mapping matrix $\mathbf{W}$ is constructed with eigenvectors of $\mathbf{R}_{t}$ on its columns (so $\mathbf{W}$ has full rank as well). Second, when $\mathbf{R}_{t}$ is not a full rank matrix, the rank of $\mathbf{R}_{t}$ reduces to $L$; this implies that only $L$ eigenvectors should be used, and thus the spatial domain encoding of STF codeword should be done across $L$ dimensions instead of $N_{t}$, giving a new $\left\{\mathbf{C}_{k}\right\}_{k} \in \mathbb{C}^{L \times p}$. In this case the maximum diversity order of $N_{t} L N_{r}$ is still achievable. Third, when $L>N_{t}$, the eigenbeamformer outputs only $N_{t}$ beams, which results in a diversity order of $N_{t}^{2} N_{r}$, and thus incurs a loss of $L-N_{t}$. So, in order to achieve the maximum diversity order, it is necessary to have $N_{t} \geq L$, or to have an eigen-weight mapping scheme that will always generate $L$ output beams.

\subsection{Coding Gain}

At a high SNR, Eqn. (6) can be expressed as $P_{r}(\mathcal{C} \rightarrow$ $\widetilde{\mathcal{C}}) \leq \gamma^{-r N_{r}} / \operatorname{det}\left(\sqrt{\mathbf{R}_{t}} \mathbf{W}^{H} \mathbf{W} \sqrt{\mathbf{R}_{t}}\right) \operatorname{det}\left(\mathbf{B B}^{H}\right)$. The coding gain can be found as $\left[\operatorname{det}\left(\sqrt{\mathbf{R}_{t}} \mathbf{W}^{H} \mathbf{W} \sqrt{\mathbf{R}_{t}}\right) \operatorname{det}\left(\mathbf{B B}^{H}\right)\right]^{-r}$. Since STF codewords have already been chosen, we can assume $\operatorname{det}\left(\mathbf{B B}^{H}\right)$ to be a constant. Given that $\mathbf{W}^{H} \mathbf{W}=I_{N_{t}}$, the coding gain is clearly affected by the correlation matrix. In general, it often results in coding gain penalty as shown in [8] where the performance reduction is proportional to the degree of spatial correlation. Thus, the coding gain is retained in the system considered.

From the channel capacity point of view, there is a possible technique in enhancing the AWG by loading unequal powers on the eigen-weighting matrix. It is shown in [10] that if power loading follows the waterpouring strategy, then the received SNR and the mutual information are maximized. In this case the joint use of transmit eigenbeamforming and diversity coding will not make sense anymore since this technique gives a reduction in the coding gain and the error rate performance can be achieved by using just beamforming.

\section{Imperfect AoA Estimation}

In this section, we analyze factors that can contribute to error rate performance loss, in particular the effect of imperfect estimation of AoA. It can be seen that the main exponents in the error probability bound in (6) are the coding gain, diversity order and the AWG. Therefore, we analyze the effect that imperfect AoA estimation has on these exponents.

For the remaining parts of this paper, we define $\widetilde{x}$ generally means an estimate of $x$. In case of imperfect AoA estimation for $L$ multipaths, let us denote $\widetilde{\mathbf{R}}_{t}$ as the new channel correlation matrix. It can also be expressed as $\widetilde{\mathbf{V}} \widetilde{\mathbf{D}} \widetilde{\mathbf{V}}^{H}$ where $\widetilde{\mathbf{V}}=\left[\underline{\underline{v}}_{1}, \cdots, \underline{\widetilde{v}}_{N_{t}}\right]$ is a unitary matrix with columns that are eigenvectors of $\widetilde{\mathbf{R}}_{t}$. Hence, a new eigen-weight mapping matrix can be found as $\widetilde{\mathbf{W}}=\left[\underline{\widetilde{w}}_{1}, \cdots, \underline{\widetilde{w}}_{N_{t}} \mid \underline{\widetilde{w}}_{j}=\right.$ $\left.\underline{\underline{v}}_{j}\right]$, and $\widetilde{\boldsymbol{\Psi}}=\sum_{k=1}^{N_{c}} \mathbf{F}_{k} \sqrt{\mathbf{R}_{t} \widetilde{\mathbf{W}}^{H}} \mathbf{B}_{k} \mathbf{B}_{k}^{H} \widetilde{\mathbf{W}} \sqrt{\mathbf{R}_{t}}{ }^{H} \mathbf{F}_{k}^{H}$ will 
be the effective error distance matrix.

To evaluate the effect of imperfect AoA estimation, we proceed as follows. It can be seen that as long as $\widetilde{\mathbf{R}}_{t}$ has a full rank, $\widetilde{\mathbf{W}}^{H}$ and $\widetilde{\Psi}$ will also be full rank matrices, and thus, there is no diversity order loss. Intuitively, this can be interpreted as STF codeword transmission over signal paths that are different from the true ones (which give highest path gains). Therefore, the maximum diversity order is retained. However, we would expect a loss in AWG and error probability in this case. To see the effect on the coding gain, we know that the product $\widetilde{\mathbf{W}}^{H} \widetilde{\mathbf{W}}$ is still an identity matrix because $\widetilde{W}$ is still a unitary matrix. Hence, the coding gain in the case of imperfect AoA estimation is the same as in the ideal case.

To quantify the loss in (6) due to incorrect estimation of AoA's, we define the transmission loss as

$$
\begin{aligned}
\xi & =\ln \left(\frac{P_{r}(\mathcal{C} \rightarrow \widetilde{\mathcal{C}}) \text { in the ideal case }}{P_{r}(\mathcal{C} \rightarrow \widetilde{\mathcal{C}}) \text { with imperfect AoA estimation }}\right) \\
& =\ln \left[\frac{\left(\sum_{j=1}^{L} \underline{w}_{j} \mathbf{R}_{t} \underline{w}_{j}^{H}\right)^{-r N_{r}}}{\left(\sum_{j=1}^{L} \widetilde{\widetilde{w}}_{j} \mathbf{R}_{t} \underline{\widetilde{w}}_{j}^{H}\right)^{-r N_{r}}}\right]
\end{aligned}
$$

The numerator is equivalent to $\left(\sum_{j}^{N_{t}} \mu_{j}\right)^{-r N_{r}}$. However, the eigenvectors used in the denominator are the estimated ones. Therefore, the denominator will always be greater than the numerator, result in a loss in error probability performance. In Fig. 2, we plotted loss function for a range of the AoA estimation error with different values of angular spread, assuming $N_{t}=4, N_{r}=2$, and $L=4$. The horizontal line indicates the maximum level of loss due to estimation error that is still acceptable. Thus the intersection points of this line with the curves indicate the minimum estimation accuracy required to secure a performance improvement from adopting the proposed scheme over the the existing STF coding.

\section{Numerical results}

Figure 3 shows the symbol/ bit error rate performance of the proposed Eigen-STF in OFDM systems as compared to systems that are only STF coded (STF) in correlated fading channel for $N_{r}=1$ and $N_{r}=2$. In our simulation, a maximum Doppler frequency of $100 \mathrm{~Hz}$ is applied and a ULA with $N_{t}=4$ and a spacing of $0.5 \lambda$ is employed at the transmitter. Simulation curves showed that Eigen-STF has a $3.5 \mathrm{~dB}$ gain in SNR over STF scheme in for SER and BER with $N_{r}=1$, at an error rate of $10^{-2}$. When the number of receive antennas is increased to two, the difference in SNR is approximately $3 \mathrm{~dB}$ at an error rate of $10^{-4}$. It is also evident up to $\mathrm{SNR}=20 \mathrm{~dB}$, the performance of the proposed Eigen-STF with $N_{r}=1$ is quite comparable to STF coded OFDM with $N_{r}=2$.

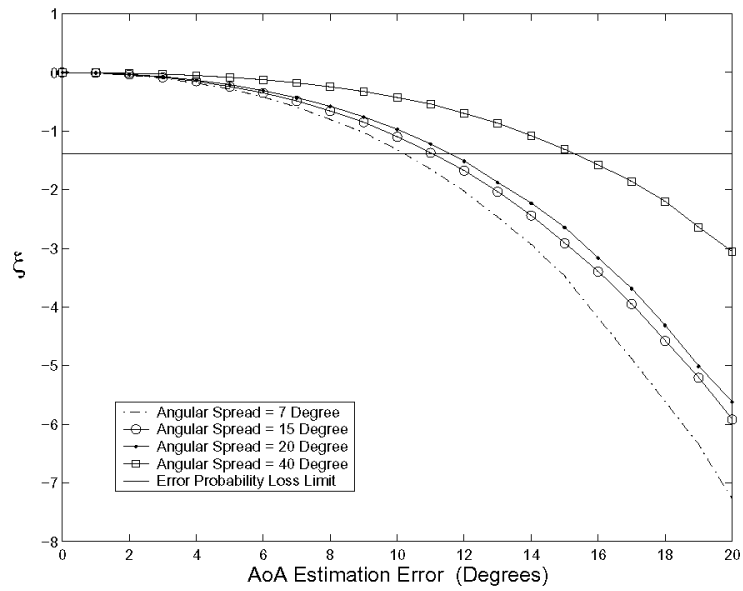

Figure 2. Transmission loss for imperfect AoA estimation as a function of AoA estimation error (in degrees) with different values of angular spread, $N_{t}=4, N_{r}=2$, and $L=4$.

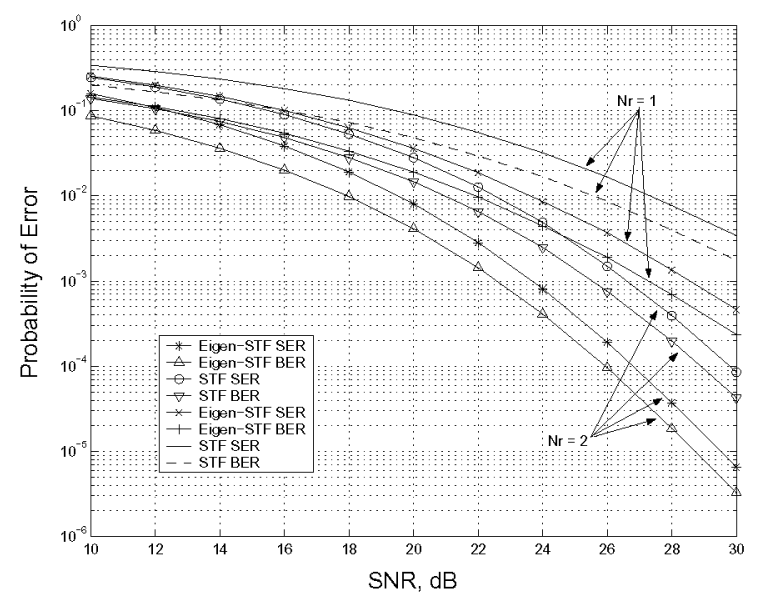

Figure 3. Error performance of OFDM systems with the proposed Eigen-STF and STF coded only transmission structures for different values of $N_{r}$ in a correlated Rayleigh fading channel with maximum Doppler frequency of $100 \mathrm{~Hz}$ and $N_{t}=4$. 


\section{Conclusions}

The performance of a new transmission scheme that combines STF coded MIMO-OFDM with adaptive eigenbeamforming is analyzed for spatially correlated frequencyselective channels. Our study proved that the diversity order and the coding gain is retained in the proposed structure while the received SNR is enhanced and the error performance is improved. In the case of imperfect AoA estimation, we quantified the performance loss due to incorrect estimations and showed that the required accuracy of estimation to secure a performance gain in different angular spreads. Simulation results also demonstrated that the proposed scheme gives better error rate performance in terms of SER and BER as compared to systems that are only STF encoded in correlated channels.

\section{References}

[1] V. Tarokh, H. Jafarkhani, and A. R. Calderbank, "Space-time block codes from orthogonal designs," IEEE Trans. Inform. Theory, vol. 45, pp. 1456-1467, Jul. 1998.

[2] V. Tarokh, N. Seshadri, and A. R. Calderbank, "Spacetime codes for high data rate wireless communication: Performance criterion and code construction," IEEE Trans. Inform. Theory, vol. 44, pp. 744-765, 1998.

[3] D. Agrawal, V. Tarokh, A. Naguib, and N. Seshadri, "Space-time coded OFDM for high data-rate wireless communication over wideband channels," in Proc. IEEE VTC, 1998, pp. 2232-2236.

[4] Y. Gong and K. B. Letaief, "Space-frequency-time coded OFDM for broadband wireless communications," in Proc. IEEE GlobeCom, Nov. 2001, pp. 519523.

[5] Z. Liu, Y. Xin, and G. B. Giannakis, "Space-timefrequency coded OFDM over frequency-selective fading channels," IEEE Trans. Signal Processing, vol. 50, pp. 2465-2476, Oct. 2002.

[6] H. Bolcskei and A. J. Paulraj, "Space-frequency coded broadband OFDM systems," in Proc. IEEE WCNC, 2000, pp. 1-6.

[7] D-S. Shiu, G. J. Foschini, M. J. Gans, and J. M. Khan, "Fading correlation and its effect on the capacity of multielement antenna systems," IEEE Trans. Commun., vol. 48, pp. 502-513, Mar. 2000.
[8] H. Bolcskei and A. J. Paulraj, "Performance of spacetime codes in the presence of spatial fading correlation," in Proc. 34th Asilomar Conf. on Signals, Systems and Computers, vol. 1, 2000, pp. 687-693.

[9] G. G. Raleigh and J. M. Cioffi, "Spatio-temporal coding for wireless communication," IEEE Trans. Commun., vol. 46, pp. 357-366, 1998.

[10] E. G. Larsson and P. Stoica, Space-Time Block Coding for Wireless Communications, Cambridge, U.K.: Cambridge Univ. Press, 2003.

[11] Siemens, Channel Model for Tx Diversity Simulations using Correlated Antennas, 3GPP Document TSGRAN WG1 \#15, R1-00-1067, Berlin, Germany, Aug. 2000.

[12] J. G. Proakis, Digital Communications, New York, N.Y.: McGraw-Hill Inc., Fourth Edition, 2001. 